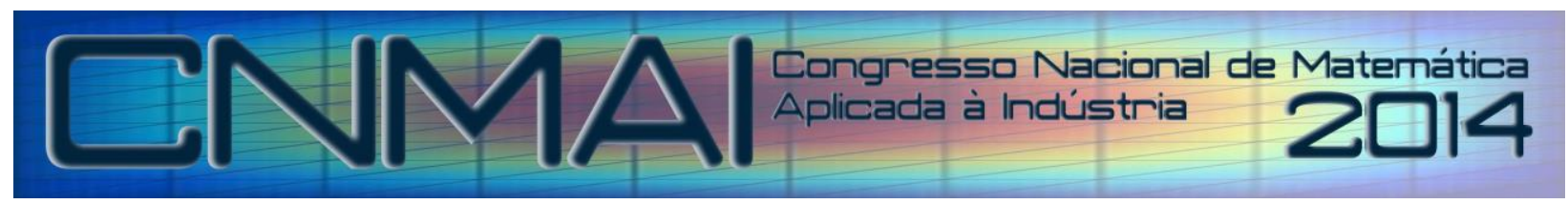

18 a 21 de novembro de 2014, Caldas Novas - Goiás

\title{
APLICABILIDADE DA METODOLOGIA CLÁSSICA PARA DETERMINAÇÃO DO COMPORTAMENTO DO FLUXO DE CALOR EM CHAPAS SOLDADAS COM DIFERENTES ESPESSURAS ATRAVÉS DAS TAXAS DE RESFRIAMENTO SIMULADAS NUMERICAMENTE
}

\author{
Rubelmar Maia de Azevedo Cruz Neto, rubelmar.neto@usp.br ${ }^{1}$ \\ Antonio do Nascimento Silva Alves, ansa@usp.br ${ }^{1}$ \\ Dario Magno Batista Ferreira, dariomagno@usp.br ${ }^{1}$ \\ Sérgio Duarte Brandi, sebrandi@usp.br ${ }^{1}$ \\ ${ }^{1}$ Universidade de São de Paulo - Departamento de Engenharia Metalúrgica e de Materiais - Av. Prof. Mello Moraes n. \\ 2463, CEP- 05508-030- Cidade Universitária - São Paulo - SP - Brasil.
}

\begin{abstract}
Resumo: Em processos de soldagem é essencial a obtenção do comportamento do fluxo de calor em chapas, isto é, se este é bidimensional (2D) ou tridimensional (3D), para um determinado conjunto de parâmetros de soldagem (tensão, corrente, velocidade de soldagem e etc). Em alguns casos, um fluxo de calor $3 D$ não é desejável, porque proporciona taxas de resfriamento máximas na região da junta soldada, induzindo a transformações de fases indesejadas. No livro Welding Handbook é apresentada uma metodologia para se determinar o comportamento do fluxo de calor, onde se obtém o valor de um parâmetro adimensional. Dependendo do intervalo onde este se apresenta, pode-se definir se o fluxo é 2D ou 3D. O objetivo deste trabalho é averiguar se este método, amplamente utilizado em aplicações industriais, por sua simplicidade e rapidez, pode ser utilizado para diferentes condições de soldagem. Foram obtidos campos de temperatura e taxas de resfriamento, através do modelo de Rosenthal para uma chapa semi-infinita de espessura finita em regime quase-estacionário. Os resultados obtidos através de simulações computacionais indicam que esta metodologia clássica nem sempre fornece informações precisas acerca do comportamento do fluxo de calor em chapas soldadas.
\end{abstract}

Palavras-chave: soldagem, simulação numérica, transferência de calor, taxas de resfriamento

\section{INTRODUÇÃO}

A soldagem a arco elétrico é um dos casos em que a transferência de calor é bastante localizada e os gradientes térmicos são bastante elevados. Consequentemente, apresenta também elevadas taxas de aquecimento e de resfriamento, afetando diretamente as microestruturas do material e, por conseguinte, alterando as propriedades mecânicas na junta soldada (Wainer et al., 1992).

As transformações metalúrgicas ocorridas tanto na ZAC como na zona fundida, em processos de soldagem, são determinadas principalmente pela taxa de resfriamento a partir da temperatura de pico alcançada durante o ciclo térmico. Taxas de resfriamento são particularmente importantes para os aços tratados termicamente, principalmente para os casos em que pode ocorrer a formação do microconstituinte martensita. Nestes casos, esta taxa crítica é muitas vezes próxima às taxas de resfriamento encontradas em processos de soldagem (American Welding Society, 2001). Adams (1958) afirma ainda que, para os aços, as microestruturas geradas são dependentes tanto da temperatura de pico alcançada no ciclo térmico, quanto do tempo de permanência em determinada faixa de temperatura, na etapa de resfriamento da solda.

Rosenthal (1941) e Adams (1958) derivaram equações para o cálculo da taxa de resfriamento, que ocorre na linha central do cordão de solda, para os casos onde o fluxo de calor na chapa é bidimensional ou tridimensional. Entretanto, é necessário conhecer o comportamento do fluxo de calor na chapa para escolher corretamente qual equação utilizar para o cálculo da taxa de resfriamento. 
Uma chapa pode ser considerada uma chapa grossa quando o fluxo de calor na soldagem é tridimensional (3D), voltado para baixo, bem como lateralmente, a partir da poça de fusão (American Welding Society, 2001). De acordo com Rosenthal (1941), uma chapa grossa passa a ser considerada infinita na espessura, a temperatura na superfície oposta à superfície soldada é a temperatura inicial da chapa. Neste modelo de chapa, as taxas de resfriamento são máximas na região da junta soldada. Dependendo da intensidade, estas taxas podem propiciar a formação de fases indesejadas no aço, como é o caso da formação de martensita, que pode vir a fragilizar bastante o material. O modelo matemático para solução de transferência de calor em uma chapa grossa (espessura infinita) utiliza uma fonte puntiforme e foi desenvolvido pela primeira vez por Rosenthal (1941), que realizou experimentos para a validação do modelo proposto.

Em constraste, durante a soldagem, uma chapa pode ser considerada como chapa fina quando o fluxo de calor a partir da poça de fusão é essencialmente lateral (2D), isto é, quando a diferença de temperatura é pequena entre as superficíes superior e inferior, em comparação com a temperatura de fusão do metal (American Welding Society, 2001). Adicionalmente, apresenta também espessura finita e o fluxo de calor é nulo na direção da espessura da chapa. A taxa de resfriamento é dependente da espessura, diferentemente do que ocorre na chapa grossa. O modelo matemático para a solução da transferência de calor em uma chapa fina (espessura finita) utiliza uma fonte de calor linear que possui comprimento igual ao da espessura da chapa, também foi desenvolvido por Rosenthal (1941). Este modelo utiliza funções modificadas de Bessel para a obtenção da solução, o que gera um aumento de complexidade para a obtenção da solução em comparação ao modelo de chapa grossa. As diferenças entre os fluxos de calor numa chapa fina e numa chapa grossa estão representados esquematicamente na Fig. 1 (American Welding Society, 2001).

Entretanto, há casos em que o fluxo de calor na soldagem não permite que a chapa seja definida como fina ou grossa. Diz-se então que a chapa é uma chapa intermediária, que possui uma espessura finita, e as isotermas não são totalmente perdendiculares à face soldada (como no caso da chapa fina), ou seja, possuem fluxo de calor nas três dimensões, próxima à zona de ligação (ZDL), que vai fazendo uma transição para fluxo de calor bidimensional quando se afasta da fonte de calor. O modelo para solução destas chapas foi desenvolvido pela a primeira vez por Rosenthal (1941), sendo adimensionalizado posteriormente por Christensen et al. (1965). Este modelo utiliza uma série de fontes imaginárias para a obtenção da distribuição de temperaturas, cuja precisão depende da quantidade de termos (ou fontes) utilizados. Este modelo é capaz de calcular gradientes de temperatura que simulam comportamentos que vão desde chapas finas até chapas grossas. Portanto, é considerado um modelo de maior robustez (GRONG, 1997).

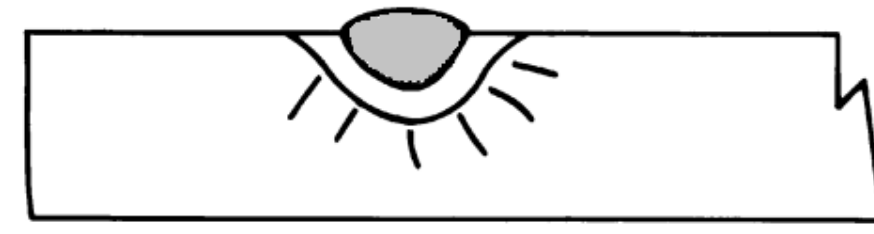

\section{Chapa Grossa \\ Fluxo de Calor 3D}

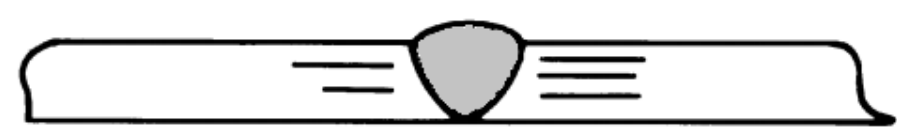

\section{Chapa Fina}

Fluxo de Calor 2D

\section{Figura 1. Fluxos de calor em chapas finas e grossas (Adaptado da American Welding Society, 2001).}

No livro Welding Handbook da American Welding Society (2001) é apresentada uma metodologia para se conhecer o comportamento do fluxo de calor na chapa antes da realização do processo soldagem, sendo a chapa classificada como fina ou grossa a partir do valor calculado de um parâmetro adimensional. Dependendo do intervalo onde este se apresenta, deve-se escolher uma ou outra equação para o cálculo da taxa de resfriamento na linha central do cordão de solda.

Dentro deste contexto, o presente trabalho tem como objetivo realizar simulações computacionais utilizando o modelo de chapa intermediária. As taxas de resfriamento resultantes serão comparadas com aquelas obtidas a partir dos modelos de chapa fina e de chapa grossa. Os erros serão calculados em relação ao modelo de chapa intermediária. Propõe-se ainda verificar se a metodologia proposta pela American Welding Society (2001) e amplamente utilizada em aplicações industriais, por sua simplicidade e rapidez, fornece informações precisas para a escolha correta entre as duas equações. 


\section{MODELOS MATEMÁTICOS}

Neste tópico será apresentada a metodologia mostrada no Welding Handbook da American Welding Society (2001) para a definição do comportamento do fluxo de calor durante a soldagem. Também serão apresentadas as equações utilizadas para o cálculo das taxas de resfriamento para chapa fina, chapa grossa e chapa intermediária, cujos modelos foram desenvolvidos por Rosenthal (1941).

\subsection{Cálculo da taxa de transferência de calor na soldagem}

Na soldagem com arco elétrico, a taxa de resfriamento $\left({ }^{\circ} \mathrm{C} / \mathrm{s}\right)$ para uma chapa grossa, na linha central do cordão de solda, foi derivada do modelo para uma chapa de espessura infinita por Rosenthal (1941), podendo ser calculada através de

$$
R_{c}^{3 D}=-\frac{2 \pi k\left(T_{c}-T_{0}\right)^{2}}{E}
$$

onde $k$ é a condutividade térmica $\left(W / m m .{ }^{\circ} \mathrm{C}\right), T_{c}$ é a temperatura de interesse ou crítica $\left({ }^{\circ} \mathrm{C}\right)$ e $T_{0}$ é a temperatura inicial ou de pré-aquecimento da chapa $\left({ }^{\circ} \mathrm{C}\right)$. $E$ é a energia de soldagem $(\mathrm{J} / \mathrm{mm})$, obtida segundo a equação:

$$
E=\eta \frac{U I}{v}
$$

onde $v$ é a velocidade de soldagem $(\mathrm{mm} / \mathrm{s}), U$ a tensão do arco elétrico $(V), I$ é a corrente (A) e $\eta$ é a eficiência de transferência de calor do arco elétrico (fonte de calor) para a chapa.

Na soldagem com arco elétrico, a taxa de resfriamento para uma chapa fina na linha central do cordão de solda foi derivada do modelo para uma chapa de espessura finita de Rosenthal (1941) por Adams (1958), podendo ser expressa por

$$
R_{c}^{2 D}=-2 \pi k \rho C\left(\frac{h}{E}\right)^{2}\left(T_{c}-T_{0}\right)^{3}
$$

onde $h$ é a espessura da chapa $(\mathrm{mm}), \rho$ a massa específica do metal de base $\left(\mathrm{kg} / \mathrm{mm}^{3}\right)$ e $C$ o calor específico do metal de base $\left(\mathrm{J} / \mathrm{kg} .{ }^{\circ} \mathrm{C}\right)$.

A taxa de resfriamento de qualquer processo de soldagem que consista de um único passe com penetração total pode ser calculada através da Eq. (3) de chapa fina (American Welding Society, 2001). No entanto, nem sempre é fácil identificar se a chapa é grossa ou fina através do número de passes, pelo fato de haver penetração total ou não, ou pela própria espessura da chapa. Por este motivo, é útil definir um parâmetro adimensional $\tau$, conhecido como espessura relativa $\tau$, obtido através de

$$
\tau=h \sqrt{\frac{\rho C\left(T_{c}-T_{0}\right)}{E}}
$$

De acordo com o procedimento descrito pela American Welding Society (2001), a Eq. (1) deve ser empregada nos cálculos da taxa de resfriamento quando o valor de $\tau$ é maior do que 0,9, enquanto que a Eq. (3) se aplica quando $\tau$ é inferior a 0,6. Quando seu valor recai entre 0,6 e 0,9, encontra-se uma região de transição entre os modelos de chapa fina e de chapa grossa. É proposta uma divisão arbitrária de $\tau$ em 0,75 , de tal modo a empregar o modelo de chapa fina para valores inferiores a este, e o modelo de chapa grossa, em caso contrário. Para esta simplificação, o erro obtido não excederia $15 \%$.

Signes (1972) reapresenta os resultados obtidos por Jhaveri et al. (1962) no gráfico da taxa de resfriamento adimensional $\kappa$ em função da espessura relativa elevada ao quadrado $\tau^{2}$.

O valor de $\tau$ indicado por Signes (1972) (Fig. 2) para a transição da chapa fina para intermediária é de aproximadamente 0,55 e para a transição da chapa intermediária para grossa é de aproximadamente 1,11, sendo estes valores obtidos através do cálculo das raízes quadradas dos valores constantes na abcissa do gráfico da Fig. 2. Enquanto que os valores de $\tau$ indicados para as mesmas transições pela American Welding Society (2001) são, respectivamente, iguais a 0,6 e 0,9 . 


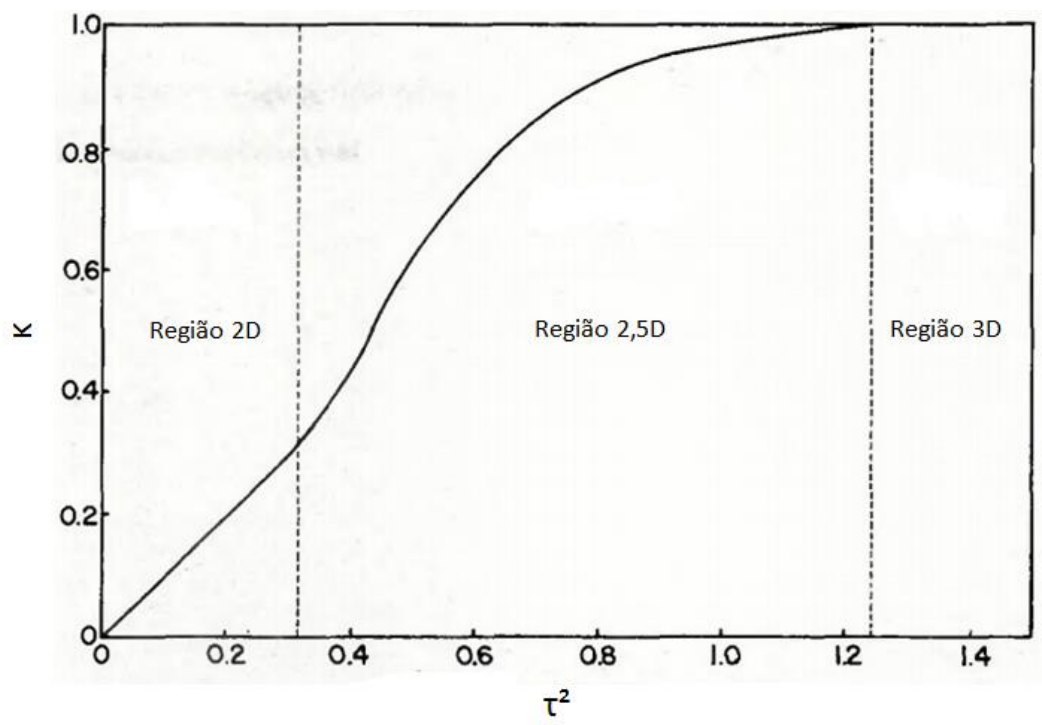

Figura 2. Razão entre as taxas de resfriamento $\boldsymbol{\kappa}$ versus espessura relativa $\tau$ (Signes, 1972).

\subsection{Modelo de Rosenthal para uma chapa intermediária}

O modelo para a solução da transferência de calor em uma chapa intermediária, desenvolvido por Rosenthal (1941) a partir da solução da equação de Fourier da transferência de calor, permite descrever os ciclos térmicos e isotermas dos diversos processos de soldagem. Este modelo considera o efeito da condução de calor e despreza os efeitos da radiação e da convecção. Consequentemente, a perda de calor pelas superfícies da chapa é desprezível. Deste modo, adota-se uma fonte puntiforme que se move em linha reta com velocidade constante e fontes imaginárias dispostas simetricamente em relação à posição da fonte real, a uma distância 2id, conforme a Fig. 3 (Myhr e Grong, 1990; Grong, 1997). Este método baseia-se no método das imagens, onde uma fonte imaginária anula o efeito da outra simetricamente oposta.

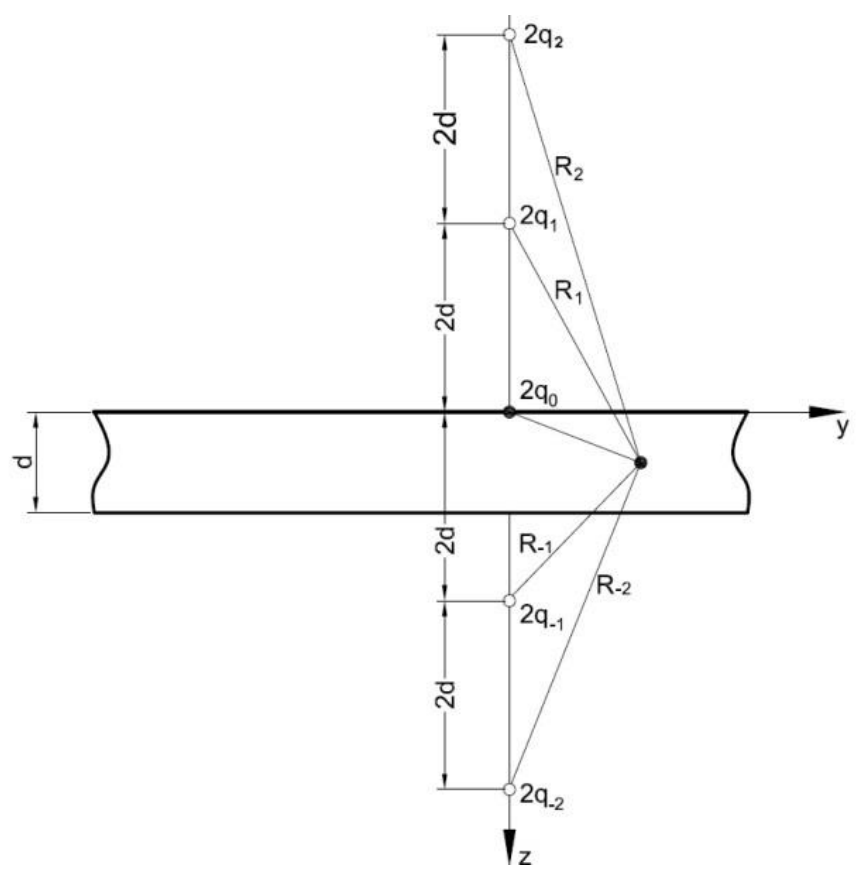

Figura 3. Disposição das fontes real e imaginárias sobre uma chapa intermediária.

De acordo com Perret (1997), para chapas grossas a espessura não exerce influência significativa sobre a forma da difusão do calor. Deste modo, o perfil das isotermas, a partir do ponto de fusão para temperaturas inferiores, varia pouco e mantém um fluxo de calor em três dimensões. Por outro lado, em chapas de espessura finita, a influência da espessura é significativa, de tal modo que o fluxo de calor pode variar seguindo um comportamento que varia entre uma chapa fina (fluxo 2D) e uma chapa intermediária (fluxo 3D). A Figura 4 ilustra a diferença entre uma chapa 
intermediária, onde a espessura é finita, e uma chapa grossa onde a espessura passa a ser infinita, ou seja, a fonte de calor não "enxerga" a face inferior da chapa.

(a) Fonte de calor em um sólido infinito

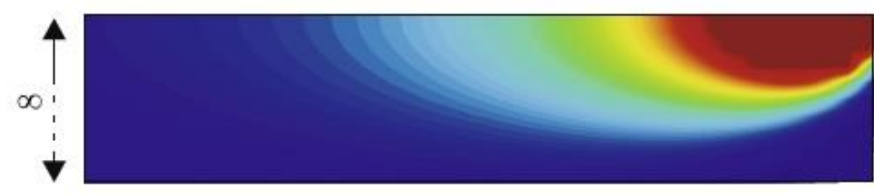

Temperatura em ${ }^{\circ} \mathrm{C}$

(b) Fonte de calor em um sólido com espessura finita $\delta$

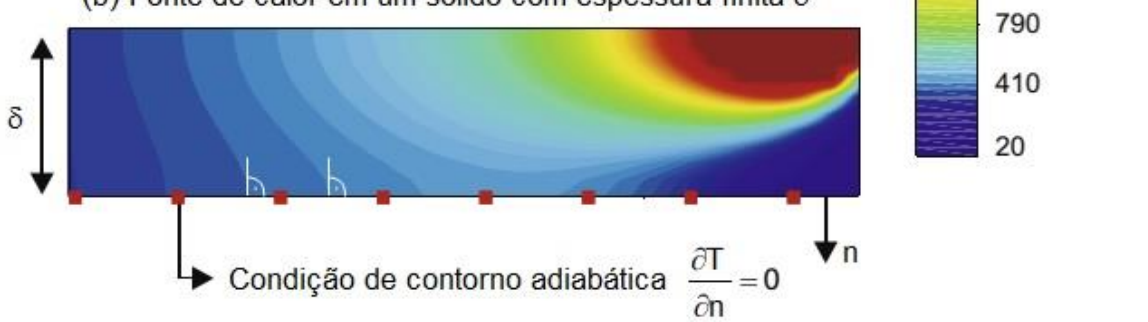

Figura 4. Vista lateral apresentando a distribuição de temperaturas durante a soldagem numa chapa grossa (a) e numa chapa intermediária (b) (Perret et al., 2010).

As Eq. (5a) e (5b) são a solução de Rosenthal para o modelo de chapa intermediária, e fornecem informações sobre a distribuição de temperatura em um regime pseudoestacionário, em todas as direções (Rosenthal, 1941).

$$
T-T_{0}=\frac{q_{0}}{2 \pi \lambda} \exp \left(-\frac{v x}{2 \alpha}\right)\left[\sum_{i=-\infty}^{i=+\infty} \frac{1}{R_{i}} \exp \left(-\frac{v}{2 \alpha} R_{i}\right)\right]
$$

onde

$R_{i}=\sqrt{x^{2}+y^{2}+(z-2 i d)^{2}}$

sendo $R_{i}$ é o vetor que tem como origem a fonte de calor em $m m, q_{0}$ é a potência do arco elétrico $(W), \alpha$ a difusividade térmica $\left(\mathrm{mm}^{2} / \mathrm{s}\right), \lambda$ a condutividade térmica $\left(\mathrm{W} / \mathrm{mm} .{ }^{\circ} \mathrm{C}\right)$ e $d$ a espessura da chapa $(\mathrm{mm})$.

Estas equações podem ser escritas, de uma forma geral, em unidades adimensionais apropriadas, conforme a Eq. (6a), que descreve a distribuição de temperaturas adimensionais em regime pseudoestacionário pode ser definido através de

$$
\frac{\theta}{n}=\exp (-\xi)\left[\sum_{i=-\infty}^{i=+\infty} \frac{1}{\sigma_{i}} \exp \left(-\sigma_{i}\right)\right]
$$

onde

$$
\sigma_{i}=\frac{v}{2 \alpha} R_{i}=\sqrt{\xi^{2}+\psi^{2}+(\zeta-2 i \delta)^{2}} ; \quad \theta=\frac{\left(T-T_{0}\right)}{\left(T_{m}-T_{0}\right)} ; \quad n=\frac{q_{0} v}{4 \pi a^{2} \rho c\left(T_{m}-T_{0}\right)}
$$

sendo $\theta$ é a temperatura adimensional, $n$ é o parâmetro operacional adimensional, $\sigma_{i}$ é o raio adimensional e $T_{m}$ é a temperatura de fusão do metal de base. A espessura adimensional $\delta$ e as coordenadas adimensionais $\xi$, $\psi$ e $\zeta$ podem ser obtidas através de

$$
\begin{aligned}
\xi & =\frac{v}{2 a} x \\
\psi & =\frac{v}{2 a} y \\
\zeta & =\frac{v}{2 a} z
\end{aligned}
$$




$$
\delta=\frac{v}{2 a} d
$$

Grong (1997) afirma que, em situações reais de soldagem não há espessura de chapa definida como grossa ou fina, pois, o fluxo de calor varia continuamente à medida que se afasta da fonte de calor, conforme representado graficamente na Fig. 4b. Deste modo, as isotermas alteram seus fluxos de calor, desde 3D (isoterma de fusão) a 2D (isoterma de $T_{0}$ ), até mesmo $1 \mathrm{D}$, dependendo das condições operacionais.

Grong (1997) afirma ainda que o modelo de chapa intermediária é o mais adequado para situações práticas de soldagem, para prever a distribuição de temperaturas de pico e levantamento de ciclos térmicos, dentro da região da ZAC, para diversas condições operacionais de soldagem, e diversos materiais, como por exemplo, alumínio (alta difusividade térmica) e aço (baixa difusividade térmica).

\section{DADOS DE ENTRADA}

As equações apresentadas no item 2 deste estudo foram implementados em um programa elaborado no software Wolfram Mathematica 9.0. Como dados de entrada para o programa foi utilizada uma chapa de aço de baixa liga com espessura variando de 5 até $50 \mathrm{~mm}$, que se encontrava a uma temperatura inicial $T_{0}$ igual a $20^{\circ} \mathrm{C}$, cujas propriedades térmicas são: o produto da massa específica pelo calor específico $\rho c=0,005 \mathrm{~J} / \mathrm{mm}^{3}{ }^{\circ} \mathrm{C}$, a difusividade térmica $\alpha=5$ $\mathrm{mm}^{2} / \mathrm{s}$ e temperatura de fusão $T_{m}=1520{ }^{\circ} \mathrm{C}$.

A Tabela 1 apresenta os parâmetros de soldagem utilizados nas simulações com o modelo de chapa intermediária. Os parâmetros foram escolhidos de forma a se ter os valores da $E$ de $0,8,1,2$, e 1,6 kJ/mm. Foi simulado o processo de soldagem MIG, sendo admitido o valor da eficiência de transferência de calor, $\eta$, igual a 0,80 .

Tabela 1. Parâmetros de soldagem utilizados nas simulações

\begin{tabular}{c|c|c|c|c|c|c}
\hline Séries & $U(\mathrm{~V})$ & $I(\mathrm{~A})$ & $\eta$ & $q_{0}(\mathrm{~W})$ & $v(\mathrm{~mm} / \mathrm{s})$ & $E(\mathrm{~kJ} / \mathrm{mm})$ \\
\hline 1 & 25 & 160 & 0,8 & 3200 & 4 & 0,8 \\
\hline 2 & 30 & 200 & 0,8 & 4800 & 4 & 1,2 \\
\hline 3 & 32 & 250 & 0,8 & 6400 & 4 & 1,6 \\
\hline
\end{tabular}

\section{RESULTADOS}

A Figura 5 exibe as isotermas de $1520^{\circ} \mathrm{C}, 1000^{\circ} \mathrm{C}, 700^{\circ} \mathrm{C}$ e $500^{\circ} \mathrm{C}$ calculas para uma energia de soldagem igual a $1,2 \mathrm{~kJ} / \mathrm{mm}$ e espessura igual $23 \mathrm{~mm}$, nestas condições esta chapa é uma chapa grossa e a taxa de resfriamento é máxima. Nenhuma das isotermas atinge a superfície inferior da chapa, neste caso a fonte de calor não "enxerga" a superfície inferior, sendo a espessura considerada infinita. Para os mesmos valores de energia de soldagem e propriedades físicas as isotermas não sofreriam alteração de forma com o aumento da espessura da chapa. Por outro lado, apenas diminuindo o valor da espessura para 14,5 mm a chapa se torna uma chapa intermediária (Fig 6).

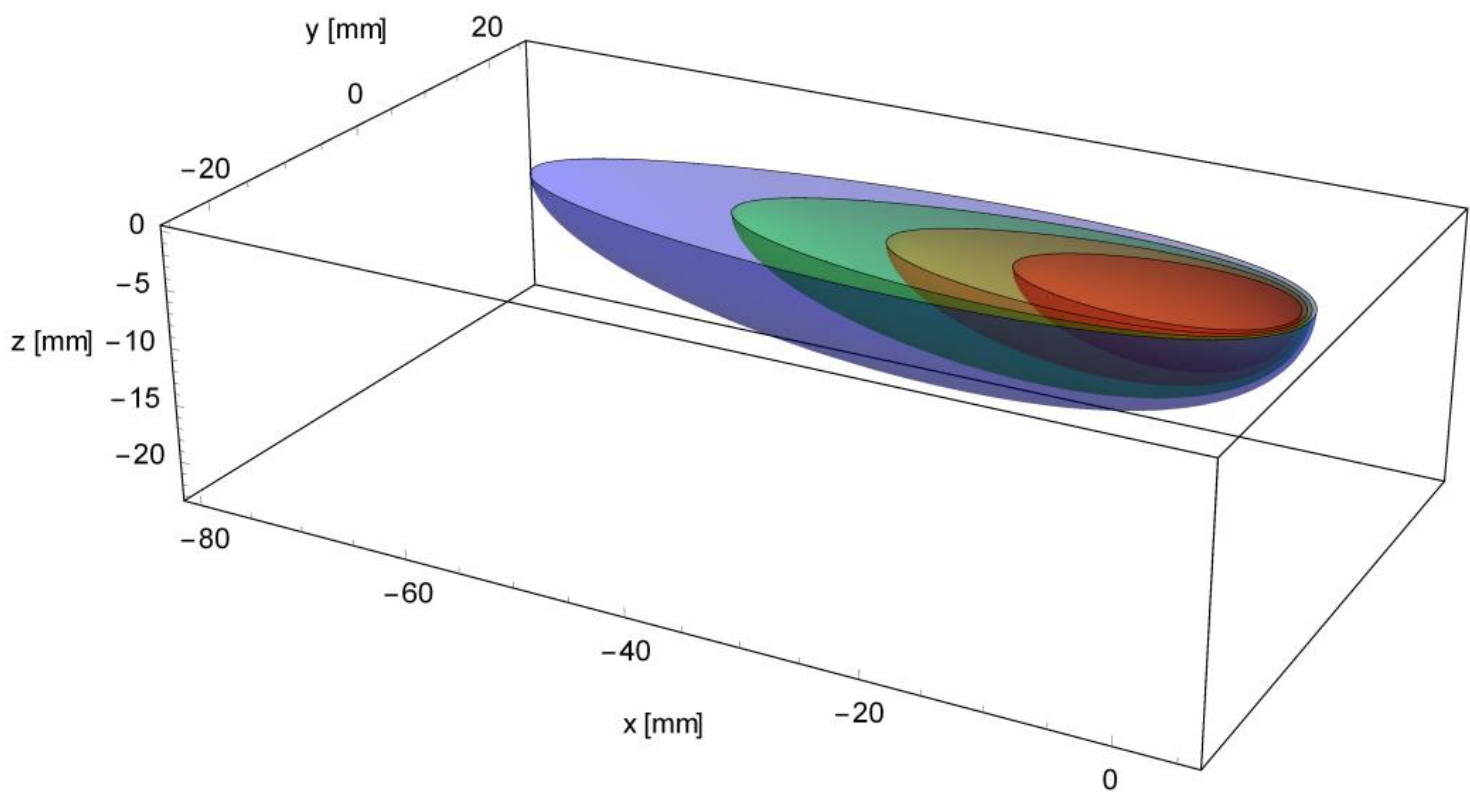

Figura 5. Isotermas em 3D para $h=23 \mathrm{~mm}$ e $E=1,2 \mathrm{~kJ} / \mathrm{mm}$. 
A Figura 7 apresenta as curvas das taxas de resfriamento $\left(R_{c}\right)$ obtidas através dos diferentes modelos de transferência de calor, em função da espessura da chapa. Pode-se observar que a curva referente ao modelo de chapa intermediária coincide com a curva da chapa fina, para os valores de $h$ entre 5 e $10 \mathrm{~mm}$ aproximadamente. Da mesma maneira, os valores de $R_{c}$ a curva de chapa intermediária coincide com os valores obtidos do modelo de chapa grossa, para uma espessura maior ou igual a aproximadamente $23 \mathrm{~mm}$.

Vale atentar que, a Eq. (1) do modelo de chapa grossa não depende da espessura da chapa, portanto, obtêm-se valores constantes para diferentes valores de espessura. $\mathrm{O}$ comportamento dos diferentes modelos está em conformidade aos resultados apresentados por Adams (1958).

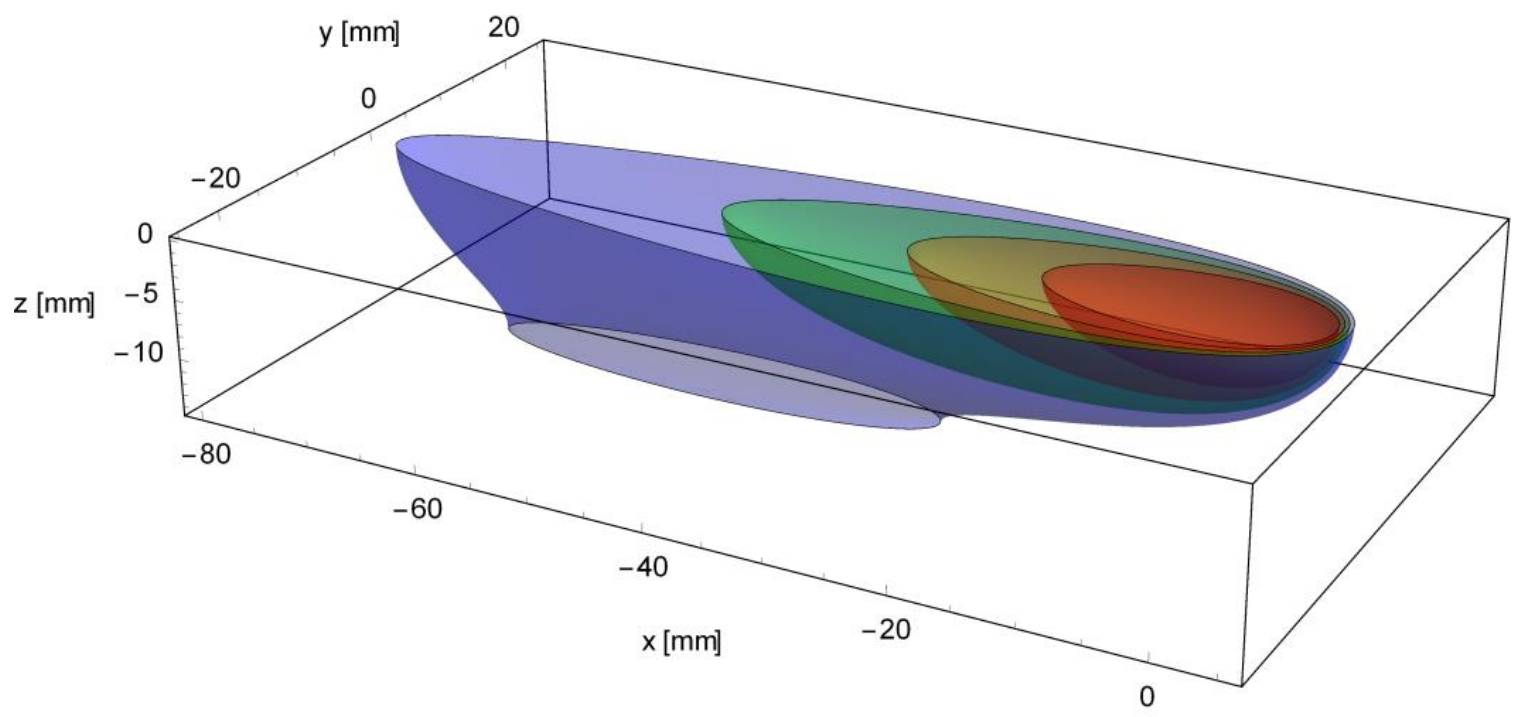

Figura 6. Isotermas em 3D para $h=14,5 \mathrm{~mm}$ e $E=1,2 \mathrm{~kJ} / \mathrm{mm}$.

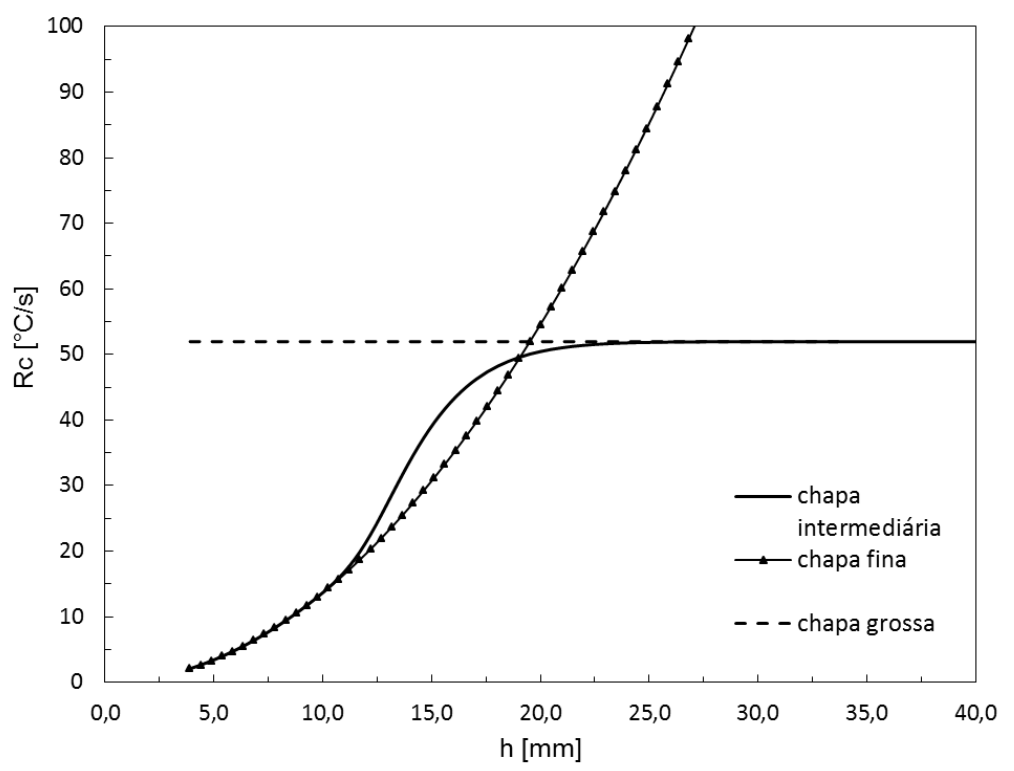

Figura 7. Taxa de resfriamento $R_{c}\left({ }^{\circ} \mathrm{C} / \mathrm{s}\right)$ versus espessura $h(\mathrm{~mm})-E=1,2 \mathrm{~kJ} / \mathrm{mm}$ e $T_{c}=650{ }^{\circ} \mathrm{C}$.

A Figura 8 exibe as curvas de erro dos modelos de chapa fina e grossa em relação ao modelo de chapa intermediária, com base na Fig. 7. Conforme citado anteriormente no item 2.2 deste trabalho, o erro máximo de se utilizar as Eqs. (1) e (3) no intervalo onde a chapa se comportaria como chapa intermediária, ou seja, para valores de $\tau$ entre 0,6 e 0,9, não excederia 15\% de acordo com a American Welding Society (2001). Pode-se verificar que o erro máximo alcançado entre o modelo de chapa fina e o de chapa intermediária (Fig. 8a) até o valor de $\tau$ igual a 0,75 fica próximo de $25 \%$, e para valores acima de 0,75 pode-se observar um erro máximo próximo de $30 \%$ entre os modelos de chapa grossa e chapa intermediária (Fig. 8b). Os erros máximos de se utilizar as Eqs. (1) e (3) para os cálculos da taxa de resfriamento em relação aos valores obtidos através da Eq. (6a), no intervalo de $\tau$ onde a chapa se comportaria como chapa intermediária, ficaram próximos aos relatados por Adams (1958) que alega que estes ficariam em torno de $25 \%$. 
Vale atentar que, os valores de erro apresentados na Fig. 8a se aproximam de zero próximo ao valor de $\tau$ de 1,0, ponto onde as curvas da chapa fina e da chapa intermediária se cruzam no gráfico da Fig. 7, próximo ao valor de 19 $\mathrm{mm}$. A partir deste ponto o erro aumenta indefinidamente, deixando evidente que erros elevados poderiam ser obtidos utilizando a Eq. (3) do modelo de chapa fina para um valor espessura onde a chapa fosse de fato uma chapa grossa.

Pode-se observar na Fig. 9 que as curvas da taxa de resfriamento adimensional em função de $\tau$ não variaram para os diferentes valores de $E$ e $T_{c}$ simulados neste estudo e, consequentemente, pode-se definir os valores de $\tau$ para caracterizar de forma generalizada a transição entre os diferentes tipos de fluxo de calor.

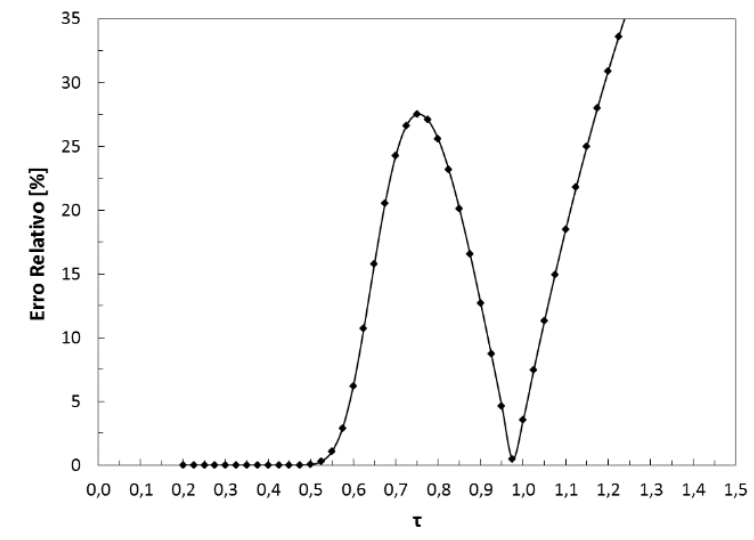

$\mathbf{a}$

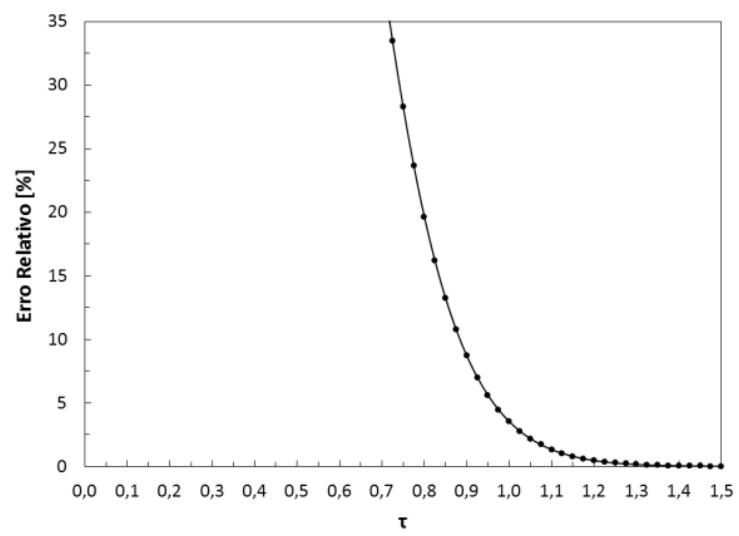

b

Figura 8. Erro da chapa fina em relação à chapa intermediária (a) e erro da chapa grossa em relação à chapa intermediária (b).

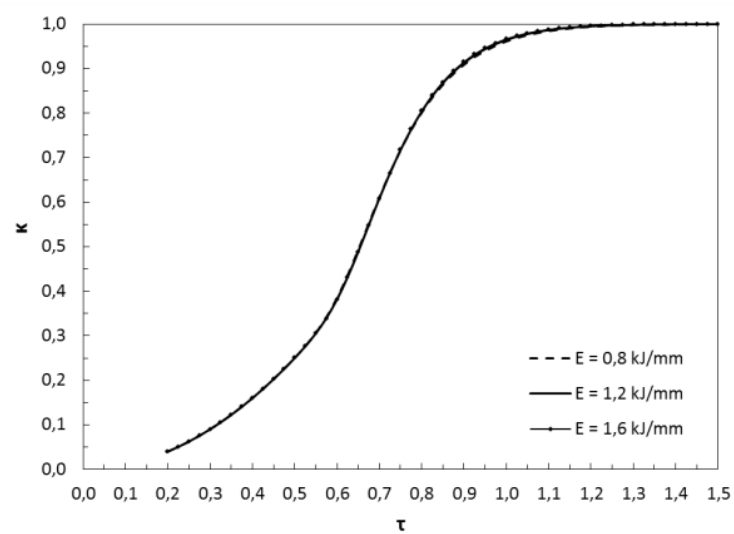

a

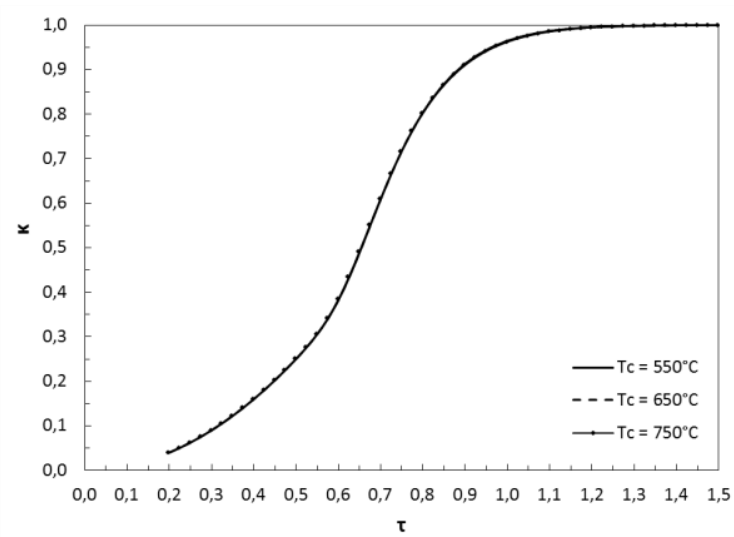

b

Figura 9. Taxa de resfriamento adimensional $\boldsymbol{\kappa}$ versus espessura relativa $\tau$ da chapa para diversos valores de energia de soldagem $E$ e b) para diversos valores de temperatura crítica $T_{c}$.

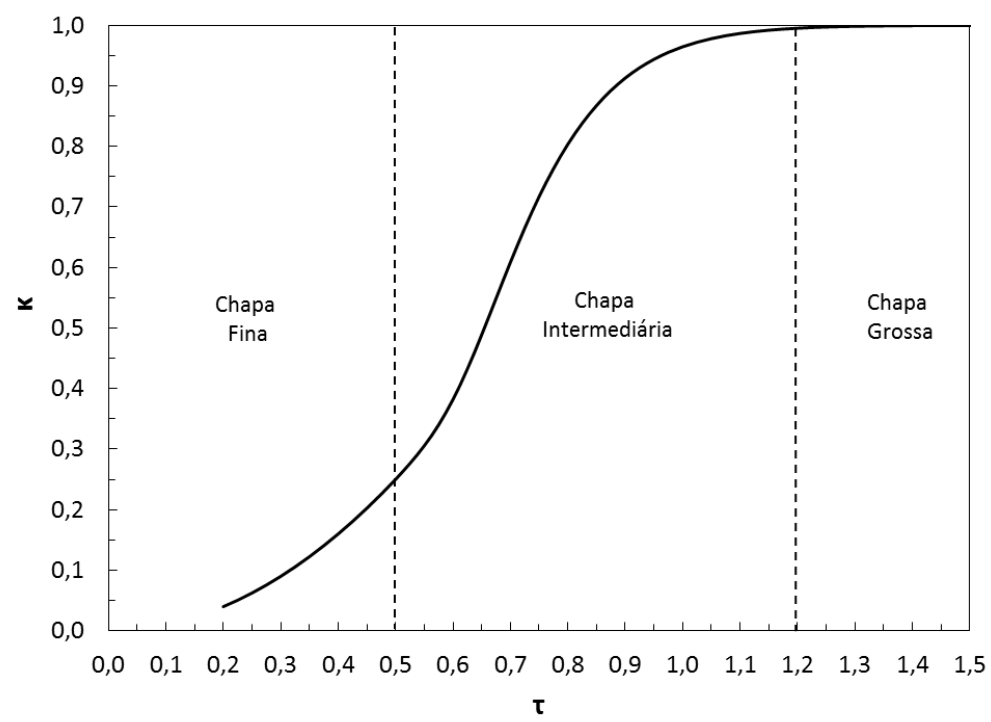

Figura 10. Transições entre os diferentes tipos de fluxo de calor na soldagem obtidos neste estudo. 
A Figura 10 apresenta os valores de $\tau$ para as transições entre os diferentes fluxos de calor na soldagem, sendo estes obtidos através das curvas de erro entre os diferentes modelos (Fig. 8). A transição da chapa fina para chapa intermediária foi obtida adotando-se como critério erros abaixo de $1 \%$, sendo encontrado um valor de $\tau$ igual a 0,52 . Acima deste valor de $\tau$, a diferença entre os valores obtidos com os dois modelos aumentam significativamente. Da mesma forma, foi definido o valor de $\tau$ para a transição da chapa intermediária para chapa grossa igual a 1,18, sendo adotados, neste estudo, os valores de $\tau$ para as duas transições iguais a 0,5 e 1,2.

Vale ressaltar que, os valores de $\tau$ obtidos neste estudo para as duas transições diferem dos valores encontrados na literatura, mas se apresentam mais próximos dos valores sugeridos por Signes (1972), que indicou estes como sendo próximos de 0,55 e 1,11. Já os valores de $\tau$ indicados pela American Welding Society (2001), iguais a 0,6 e 0,9, diferem bastante aos encontrados neste trabalho.

\section{CONCLUSÕES}

As simulações realizadas neste estudo utilizaram o valor de erro de convergência da solução do modelo de chapa intermediária de $10^{-6}$ para a obtenção de uma solução precisa, e o número de fontes imaginárias variou de 7 até 29 para garantir a precisão desejada, sendo utilizado o método de Newton-Raphson para o cálculo das raízes das funções.

Como se pode observar, os valores de $\tau$ indicados para definir o tipo de fluxo de calor, diferem entre Signes (1972) e da American Welding Society (2001), assim como também diferem dos valores obtidos neste trabalho. Esta divergência entre os valores $\tau$ obtidos neste trabalho pode ser proveniente do elevado número de fontes imaginárias utilizadas para a convergência da solução, o que acarretou em uma precisão também bastante elevada nos valores obtidos das temperaturas e das taxas de resfriamento. Isto reforça a afirmação de Grong (1997) a respeito da robustez do modelo de chapa intermediária, pois os valores de $\tau$ se distanciaram ainda mais neste trabalho.

Analisando-se as curvas de erro obtidas neste estudo se torna evidente que a utilização indevida do modelo de chapa fina para o caso da chapa ser uma chapa grossa, ou seja, para valores de $\tau$ maiores que 1,2, pode propiciar erros extremamente elevados. Ressaltando a importância de se conhecer previamente o comportamento do fluxo de calor na soldagem para a escolha correta da equação para o cálculo da taxa de resfriamento.

Adotando-se a metodologia apresentada pela American Welding Society (2001) para a escolha da equação da taxa de resfriamento a ser utilizada no intervalo de $\tau$ de 0,6 a 0,9 foi obtido um erro próximo $25 \%$, enquanto esta afirmava que o erro não ultrapassaria os $15 \%$. Portanto, isto sugere a utilização do modelo de chapa intermediária para o cálculo dos ciclos térmicos, distribuição de temperaturas e taxas de resfriamento. Todavia, a utilização do modelo de chapa intermediária requer maior tempo de modelagem do problema e custo computacional em relação aos outros modelos.

\section{AGRADECIMENTOS}

Os autores gostariam de agradecer ao Programa de Pós-Graduação em Engenharia Metalúrgica e de Materiais da USP - Universidade de São Paulo, à FAPEAM- Fundação de Amparo à Pesquisa do Estado do Amazonas (R. M. A. C. N. e A. N. S. A.), à CAPES- Coordenação de Aperfeiçoamento de Pessoal de Nível Superior e ao IFES - Instituto Federal de Educação, Ciência e Tecnologia do Espírito Santo (D. M. B. F.) pelo apoio financeiro.

\section{REFERÊNCIAS}

American Welding Society. 2001. Welding Handbook. 9. Edição: Cynthia L. Jenney e Annette O'Brien. Vol. 1. Miami, Florida.

Adams,C. M. Jr. 1958. Cooling Rates and Peak Temperatures in Fusion Welding. Welding Journal, 37(5), 210-215.

Christensen, N., Davies, V. L., Gjermundsen, K. 1965. Distribution of temperatures in arc welding. Britsh Welding

Journal, 54-75

Grong, Oisten. 1997. Metallurgical Modelling of Welding. 2a . Trondhein: The University Press.

Jhaveri , P., Moffatt, W. G., and Adams, C .M, Jr. 1962. The Effect of Plate Thickness and Radiation on Heat Flow in Welding and Cutting. Welding Journal, 41(1), 12-16.

Myhr, O. R., e O. Grong. 1990. Dimensionless maps for heat flow analyses in fusion welding. Acta Metallurgica et Materialia, 449-460.

Perret, W., Schwenk, C., Rethmeier, M. 2010. Comparison of analytical and numerical welding temperature field calculation. Computational Materials Science, 47, 1005-1015.

Rosenthal, D. 1941. Mathematical Theory of Heat Distribution during Welding and Cutting. Welding Journal, 20(5), 220-234.

Signes, E. G. 1972. A Simplified Method for Calculating Cooling Rates in Mild and Low Alloy Steel Weld Metals. Welding Journal, 172, 473-484.

Wainer, Emílio, Sérgio Duarte Brandi, e Fábio Decourt Homem Mello. Soldagem: Processo e Metalurgia. São Paulo: Edgard Blücher Ltda., 1992. 
Congresso Nacional de Matemática Aplicada à Indústria, 18 a 21 de novembro de 2014, Caldas Novas - GO

\section{RESPONSABILIDADE AUTORAL}

Os autores são os únicos responsáveis pelo conteúdo do material impresso incluído neste trabalho. 


\title{
APPLICABILITY OF CLASSICAL METHODS FOR DETERMINING THE HEAT FLOW BEHAVIOR IN WELDED PLATES WITH DIFFERENT THICKNESS THROUGH THE COOLING RATES NUMERICALLY SIMULATED
}

\author{
Rubelmar Maia de Azevedo Cruz Neto, rubelmar.neto@usp.br ${ }^{1}$ \\ Antonio do Nascimento Silva Alves, ansa@usp.br ${ }^{1}$ \\ Dario Magno Batista Ferreira, dariomagno@usp.br ${ }^{1}$ \\ Sérgio Duarte Brandi, sebrandi@usp.br ${ }^{1}$ \\ ${ }^{1}$ Universidade de São de Paulo - Departamento de Engenharia Metalúrgica e de Materiais - Av. Prof. Mello Moraes n. \\ 2463, CEP- 05508-030- Cidade Universitária - São Paulo - SP - Brasil.
}

\begin{abstract}
In welding processes is essential to obtain the heat flow behavior in plates, i.e., if it is two-dimensional (2D) or three-dimensional (3D) for a given set of welding parameters (voltage, current, welding speed, etc.). In some cases, $3 D$ heat flow is not desirable, because it provides maximum cooling rates in the weld zone, inducing undesired phase transformations. In the book Welding Handbook a method is presented to determine the heat flow behavior, where the dimensionless parameter value is given. Depending on the range where it presents we can determine whether the flow is $2 D$ or $3 D$. The objective of this study is to investigate if this method, widely used in industrial applications due to its simplicity and speed, can be used for different welding conditions. Temperature distribution and cooling rates are obtained by Rosenthal method to a semi-infinite plate of finite thickness in a quasi-stationary state. The results obtained through computer simulations indicate that this classical approach doesn't always provide accurate information about the heat flow behavior in welded plates.
\end{abstract}

Key-Words: welding, numerical simulation, heat transfer, cooling rates 